\title{
Fabrication of thermal sensitive folic acid based supramolecular hybrid gels for injectable drug release gels $\uparrow$
}

Yahui Song, Jianping Gao, Xiaoyang Xu, Huilin Zhao, Ruinan Xue, Jingkuo Zhou, Wei Hong and Haixia Qiu*

School of Science, Tianjin University, Tianjin 300072, China

\begin{abstract}
Thermal sensitive supramolecular hybrid gels for injectable drug release were prepared by adding different amounts of agar into folic acid (FA) gelator. The gelation temperature was modulated in order to form injectable gel with body temperature (37 ${ }^{\circ} \mathrm{C}$ ). Such kind of folic acid-agar (FAG) hybrid gel makes it possible to use supramolecular gel as injectable drug loaded gels for drug release. FT-IR and UV-vis spectra indicate that agar macromolecules involve in the self-assembly process through intermolecular H-bonding and $\pi$ - $\pi$ stacking interactions with FA molecules. The SEM and TEM images demonstrate that the fibre diameter of FAG hybrid gel is about $20 \mathrm{~nm}$, much smaller than that of FA gel (40 nm). However, FAG hybrid has a denser nano-fibrous network structure than FA gels. Moreover, FAG hybrid gel is endowed with a more ordered network structure and a little better crystallization capability by adding agar. FAG hybrid gel also shows a shear-thinning behavior but the shear viscosity is about 2 times higher than that of FA gel. Compared with FA gel, the storage $\left(\mathrm{G}^{\prime}\right)$ and loss $\left(\mathrm{G}^{\prime \prime}\right)$ moduli of the FAG gel are higher, which implies an enhanced gel strength. At the same time, both FA and FAG gels are facilely affected
\end{abstract}


by some external factors such as acid, base and salts. In acidic or basic conditions, the strength became weak and the gelation temperature $(\mathrm{Tg})$ decreased. While, within certain concentrations, the salt $(\mathrm{NaCl})$ increased the gel strength and $\mathrm{Tg}$. FAG gel suffered lower mass loss and owned better stability in different $\mathrm{pH}$ solutions compared with pure FA gel.The release behavior of the FA and injectable FAG gels was investigated by using Rhodamine $\mathrm{B}$ as a mimic model drug. FAG hybrid gel shows a long release profile and the release time is 3 times longer than that of FA gel, up to 30 hours, and the accumulative release amount reaches about $86 \%$. So it is a potential injectable gel for sustained release drug delivery system.

\section{Introduction}

Supramolecular gel, as a branch of supramolecular chemistry, has become a research hotspot in recent years. Some small molecular weight organic compounds, which are also called low molecular weight gelators (LMWGs), can gelatinize the solvents at very low concentration (even below $2 \mathrm{wt} \%$ ) via self-assembly and form supramolecular gels. ${ }^{1,2}$ Different from traditional gels which are driven by covalent bond, the driving forces of supramolecular gels are weak and reversible non-covalent interactions between two or more molecules, including hydrogen bonds, van der Waals forces, $\pi-\pi$ stacking, dipole-dipole interactions, hydrophilic/hydrophobic effects, etc. ${ }^{3,4}$ Through the synergy of these non-covalent bonds, gelators form one-dimensional ordered nanostructures, such as fibrils or tapes, which then stack to microfibers that finally twisted into three-dimensional networks. The solvent 
molecules, wrapped into the three-dimensional networks, cannot flow under gravity because of the surface tension between supramolecular aggregates and the solvents. ${ }^{5,6}$ So the gel is solid on the macroscopic and looks like hydrogel prepared from hydrophilic macromolecules. The preparation of the gel is usually a process that includes heating the mixture system to give a homogeneous supersaturated solution and then cooling it to gelation temperature ( $\mathrm{Tg}$ ) at which the solution undergo a sol to gel transition and form a gel. ${ }^{7,8}$ As the self-assembly process of supramolecualr gels is generally kinetically trapped rather than thermodynamically limited, there will be three possible states of the solution during the gel formation: i) highly ordered aggregates (namely crystal) ${ }^{9}$ ii) random aggregates (i.e. amorphous precipitate); iii) intermediate state of the first two aggregates (gel). ${ }^{10}$ Small molecular organic gels that benefit from the reversibility of the physical interactions between LMWGs have their own unique characteristics: thermoreversibility (the gel can undergo a gel to sol transition at a certain temperature, Tgel-sol), self-healing properties (the systems can spontaneously repair their damage after being broken down under certain conditions), changeable viscoelasticity, and susceptible to the external stimuli (including temperature, $\mathrm{pH}$, ionic strength, light, etc.). ${ }^{11,12}$ Thus, the supramolecular gels become highly promising candidates for a variety of applications, e.g. biomaterials, ${ }^{13,14} \mathrm{drug}$ release, ${ }^{15}$ sensors, ${ }^{16}$ pollutant removal from water, food, personal care formulations and so on.

Due to the reversibility of non-covalent bonds, the formed gels are often brittle materials with inferior mechanical properties. In addition, long-term stability is also a 
crucial issue that should be considered when supramolecular gels are put into use. So, there is a growing interest in hybrid soft gels in recent years because they demonstrate enhanced properties and good morphology. Such kind of hybrid soft gels can be made by adding different components, such as macromolecular polymers and surfactants, etc. For example, Adhia and co-workers utilize a hydrophilic polymer, poly (acrylic acid), to improve the properties of pyridine-based gels by increasing the number of crosslinked fibers. ${ }^{17}$ Priyadarshi Chakraborty and co-workers introduce chitosan into the gelator to improve the strength of the supramolecular gel and thus increase absorption capability of dyes and metal ions. ${ }^{18}$ In this article, we aim to obtain a kind of non-toxic hybrid gel that can be used in drug release system. We choose folic acid (FA, Fig.1), a vitamin B, as the gelator owing to its non-toxic and edible properties. FA plays an important role for the body to make DNA, RNA, and metabolise amino acids which are required for cell division. ${ }^{19}$ Humans cannot make it and must be required from the diet. ${ }^{20}$ Pengyao Xing and co-workers has once fabricated FA gel. ${ }^{21}$ However, the strength of pure FA gel is so weak that it severely limits its application. So, in this article, we select the agar (a kind of polysaccharide of biological macromolecular, Fig. 1) as the second component to form a FA-agar (FAG) hybrid gel in the mixed solution of DMSO-water (the volume ratio is $1: 1){ }^{22}$ Agar has a strong soliedifiability, so the molecular chains of agar could serve as supporting frames during the formation of hybrid gel. FA-agar mixture solution is temperature sensitive and can turn into FAG hybrid gel at human body temperature with different gelation time by adjusting ratio of agar and $\mathrm{NaCl}$. The FAG supramolecular gel has denser and 
stronger network structures compared with pure FA gel. So, such gelator has great potential application as injectable gel since the liquid formulations can turn into gels when they are injected and contact with human body. ${ }^{23-25}$ The present work will primarily investigate the gelation temperature, gel strength and stability, and the controlled-release pattern of a model molecule.<smiles>CCO[C@@H]1[C@H](O)C(OC2C(C)CC3OC2OC(C)[C@@H]3O)O[C@H](CO)[C@@H]1O</smiles>

FA agar

Fig. 1 Molecular structures of folic acid and agar.

\section{Experimental}

\section{Materials}

Both folic acid (FA) and agar were purchased from Aladdin-reagent Co. Ltd., Shanghai, China. Rhodamine B (RB) was purchased from Heowns Co. Ltd., Tianjin, China. The solvent dimethyl sulfoxide (DMSO, GR) and all other chemicals were purchased from Tianjin Chemical Reagent Co. Ltd.

\section{Preparation of FA, FAG hybrid gel}

FA gels were prepared as follows: $20 \mathrm{mg}$ of folic acid powder was added to $5 \mathrm{~mL}$ of DMSO at $70{ }^{\circ} \mathrm{C}$ in a glass vial under stirring. After complete dissolution, $5 \mathrm{~mL}$ of distilled water was added and then continued to stir until a transparent solution was 
formed. The mixed solution was cooled down to $30{ }^{\circ} \mathrm{C}$ and maintained at that temperature without any disturbance. The formation of the gel was recognized by inverting the glass vial shown in Fig. 2. The FAG hybrid gels were prepared by dissolving certain amounts of agar in $0.2 \%(w / v)$ FA solutions (the volume ratio of DMSO-water was still 1:1) and stirring at $70{ }^{\circ} \mathrm{C}$ until they became homogeneous. After cooling to Tg, we could observe the transparent FAG gels ( Fig. S1). The Tg of the gel was measured by immersing the glass vial containing the hot solution in a thermostated bath and lowering the temperature slowly at a rate of $0.5{ }^{\circ} \mathrm{C} \min ^{-1}$ until the gel was formed.

\section{In vitro drug release studies}

In this paper, Rhodamine B (RB) was selected as a mimic model drug to prepare RB loaded FA and injectable FAG gel since its UV absorbance peak (553 nm) has no overlaps with any other absorbance peaks of the components used in the gel. ${ }^{26}$ The RB contained gels were prepared as follows: A certain amount of RB was dissolved in distilled water to get a solution with a concentration of $5 \mathrm{mg} / \mathrm{L} .5 \mathrm{~mL}$ of such $\mathrm{RB}$ solution was mixed with $5 \mathrm{~mL}$ DMSO solution which contains FA or/and FA-agar for the preparation of FA and FAG gels. The mixture was heated at $70{ }^{\circ} \mathrm{C}$ under stirring until it formed a homogeneous solution. The hot solution was allowed to cool to get the required gel. The concentration of RB in the gel is $2.5 \mathrm{mg} / \mathrm{L}$. The normal saline solution $(0.9 \% \mathrm{NaCl})$ was chosen as the release medium. $10 \mathrm{~mL}$ of $\mathrm{RB}$ contained $\mathrm{FA}$ or FAG gels was placed in $40 \mathrm{~mL}$ release medium, respectively, and UV spectrophotometer was used to monitor the absorbance at $553 \mathrm{~nm}$ at different times. 


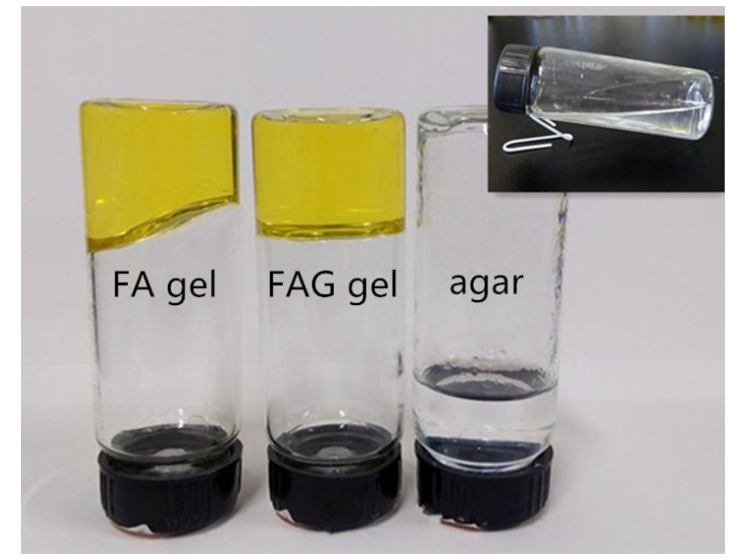

Fig. 2 FA gel, FAG hybrid gel and $0.13 \%$ (w/v) agar solution.

\section{Characterization}

The gels were frozen in liquid nitrogen and further lyophilized with a freeze dryer system under vacuum for at least $24 \mathrm{~h}$. The obtained xerogels (dried gel) were then coated with a layer of gold before they are observed with a scanning electron microscope (SEM) (Nanosem 430). The samples for transmission electron microscopy (TEM, Tecnai G2 F20) were prepared by dispersing FA or FAG xerogels in ethanol and then dropping the dispersion solutions on carbon coated copper grids, being air-dried at room temperature. Through the images of SEM and TEM, we can observe and compare the three-dimensional network structures of FA and FAG gels.

Fourier Transform Infrared (FT-IR) spectra of FA and FAG xerogels were recorded with $\mathrm{KBr}$ powder at room temperature on a Bruker ALPHA FT-IR. The position and intensity variation of the characteristic absorption peaks can be used to analyze the interactions between FA and agar molecules.

Ultraviolet-visible (UV-vis) spectra of FA solution, FA and FAG gels were 
measured with a UV-6100, China.

The crystal structure of FA and FAG hybrid gels were analyzed in the scanning range of $10-70^{\circ}(2 \theta)$ on an X-ray diffraction (XRD, D/MAX-2500, Japan).

Rheology is a useful tool to study the mechanical properties of supramolecular gels under the influence of an applied stress. The rheology studies of FA and FAG hybrid gels were investigated at $25{ }^{\circ} \mathrm{C}$ with an advanced rheometer (Stress Tech, USA).

\section{Results and discussion}

\section{Fabrication of injectable FAG gels formed at body temperature}

FA, a B vitamin, is essential for human body to synthesize DNA, repair DNA and to act as a cofactor in certain biological reactions. ${ }^{27}$ FA powder can be dissolved in DMSO, but is not soluble in water. In the mixed solution of DMSO/water (1/1, v/v), $0.2 \%(\mathrm{w} / \mathrm{v})$ of folic acid solution can form a gel at $30{ }^{\circ} \mathrm{C} .{ }^{18}$ While, the weak gel strength and unadjustable Tg greatly limit the applications of FA gel. So, in this paper, we added another component, agar, to enhance its properties. The results shown in Tab. 1 indicate that agar content has a significant influence on the Tg of FAG hybrid gels. With the increase of agar concentration the Tg of FAG hybrid gels remained rising. As the agar concentration reached $0.13 \%(w / v)$ (as shown in Fig. 2, 0.13\% agar itself is slightly viscous liquid), the $\mathrm{Tg}$ rose to $37.5{ }^{\circ} \mathrm{C}$, close to body temperature, which provided an injectable FAG solution that can transform into gel once it contacts with human body. At the same time, the structures of FAG gels were highly branched 
and became denser with the increase of agar concentration (Fig. S3, S4). When the concentration of agar exceeded $0.13 \%$, the agar solutions became viscous or even turned into gels, therefore, unless specified, $0.13 \%(w / v)$ of agar was chosen to study in the later experiments.

Tab. 1 Effect of agar concentration on $\mathrm{Tg}\left(\mathrm{C}_{\mathrm{FA}}\right.$ is $\left.0.2 \%(\mathrm{w} / \mathrm{v})\right)$

\begin{tabular}{ccccccc}
\hline $\mathrm{C}_{\text {agar }} \%(\mathrm{w} / \mathrm{v})$ & 0 & 0.025 & 0.050 & 0.075 & 0.100 & 0.130 \\
\hline $\operatorname{Tg}\left({ }^{\circ} \mathrm{C}\right)$ & 30 & 34 & 34.5 & 35 & 36.5 & 37.5 \\
\hline
\end{tabular}

The increase of Tg may be induced by the interaction between FA and agar during gel formation. So FT-IR analysis was utilized to characterize the formation process of the FA and FAG gels. Figure 3 shows the FT-IR spectra of FA and FAG xerogels. The spectrum of FA powder (Fig. 3a) presents complex vibrational peaks within the range of $3000-3600 \mathrm{~cm}^{-1}: 3544 \mathrm{~cm}^{-1}$, stretching vibration peak of -O-H in -COOH groups; 3416 and $3324 \mathrm{~cm}^{-1}$, the antisymmetric and symmetric stretching vibration (Vas, Vs) of $-\mathrm{N}-\mathrm{H}$ in $-\mathrm{NH}_{2}$ groups; $3110 \mathrm{~cm}^{-1}$, stretching vibration of $-\mathrm{C}-\mathrm{H}$ in benzene ring. ${ }^{28}$ While, the absorption peaks in the region of $3000-3600 \mathrm{~cm}^{-1}$ in FA powder become a broad band at about $3262 \mathrm{~cm}^{-1}$ in FA gel, suggesting strong interactions among FA molecules, like hydrogen bonds between carboxylic acid and amide groups. And in FAG gel, this band is shifted to $3390 \mathrm{~cm}^{-1}$ because of the molecular interactions among the FA and agar molecules. The absorption peak of $\mathrm{C}=\mathrm{O}$ in $-\mathrm{COOH}$ groups of FA powder is also moved from $1692 \mathrm{~cm}^{-1}$ to $1698 \mathrm{~cm}^{-1}$ in FA gel and $1696 \mathrm{~cm}^{-1}$ in FAG gel, indicating H-bonding interactions during the gel formation. Compared with FA powder, the disappearing of the peaks in $917 \mathrm{~cm}^{-1}$ and $838 \mathrm{~cm}^{-1}$ (the out-plane bending vibration of $-\mathrm{O}-\mathrm{H}$ in $-\mathrm{COOH}$ and $-\mathrm{N}-\mathrm{H}$ in $-\mathrm{NH}_{2}$ of FA powder, respectively) 
of FA and FAG gels implies the participation of $-\mathrm{COOH},-\mathrm{NH}_{2}$ in the process of gel formation. The amide-II bond of FA powder at $1485 \mathrm{~cm}^{-1}$ is shifted to $1514 \mathrm{~cm}^{-1}$ in FA gel and $1512 \mathrm{~cm}^{-1}$ in FAG gel. Overall intermolecular hydrogen bonds play important roles in the process of self-assembly ${ }^{29}$ and agar molecules involve in the gel formation via forming hydrogen bonds with FA molecules. According to UV-vis spectra in Fig. 4, we could easily identify the noncovalent interactions during the gel formation. The absorption peak of FA powder is at $284 \mathrm{~nm}$, but the corresponding absorption band of FA gel red-shifts by about $5 \mathrm{~nm}$ due to the $\pi-\pi$ interaction during self-assembly process. For FAG gel, it has a $10 \mathrm{~nm}$ red shift compared to FA gel. In the system of FAG gel, we think that agar acts like a polar solvent. In general, the polarity of excited state is stronger than ground state for the $\pi-\pi^{*}$ transition in polar solvent. So the interaction intensity of the excited state with the polar solvent is greater than that of the ground state, and as a result, the energy of the excited state decreases more than that of the ground state. Consequently, with the increase of the polarity of the solvent, the energy required for the transition is reduced and the absorption peak is red-shifted. The polarity of the whole FAG gel system is increased because of the $-\mathrm{OH}$ polar groups in the agar, so the FAG gel has a red shift compared to the FA gel. 

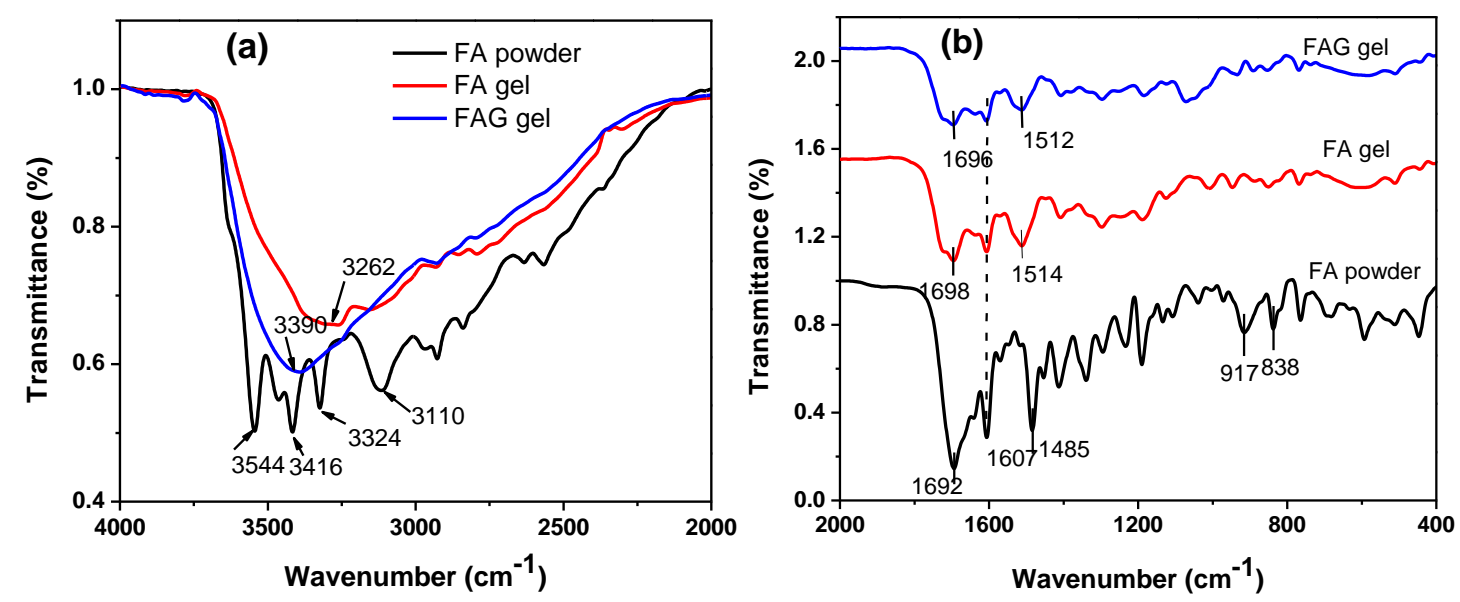

Fig. 3 FT-IR spectra of FA powder, FA and FAG xerogels.

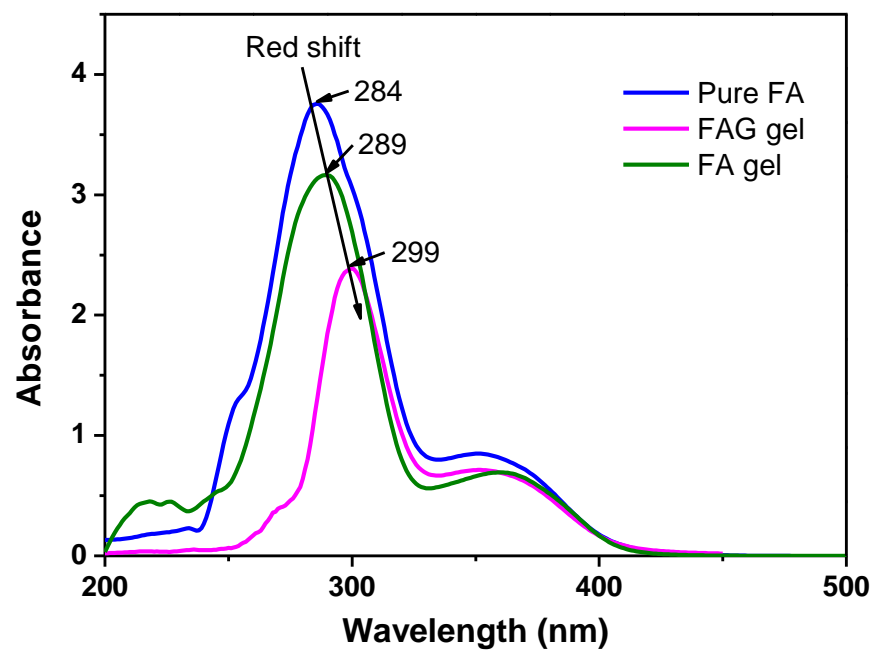

Fig. 4 UV-vis spectra of FA powder dissolved in DMSO, FA and FAG xerogels

dissolved in distilled water.

SEM observations were performed to examine the surface morphology of FA and FAG gels. The SEM images demonstrate a significantly different morphology of FA and FAG gels, as shown in Fig. 5a-b. The FA gel is composed of stubby and unordered fibers with diameters of 30-60 $\mathrm{nm}$ (most are about $40 \mathrm{~nm}$ ) and has few branched fibers. And, as the driving force of self-assembly is non-covalent interactions, the structures of FA gel are in temporary contact with each other and can be modified easily. ${ }^{30,31}$ So, FAG hybrid gel exhibits a dense and intertwined fibrillar 
network structure, although the diameters were much smaller, 20-40 nm (most are about 20 nm, Fig. 5b). Meanwhile, with the agar molecules co-localized in the fibers, the longitudinal growth of FA molecules is disrupted, and the agar molecules absorb onto the fibers and produce defect points at the fiber tips, as the crystallographic mismatch, leading to the branching of the fibers and the slowing of its growth rate. ${ }^{32}$, ${ }^{33}$ As a consequence, the FAG hybrid gel fibers are much thinner but more numerous, which generate more entangled fibers. So, the strength of hybrid gel has significantly increased compared with FA gel.

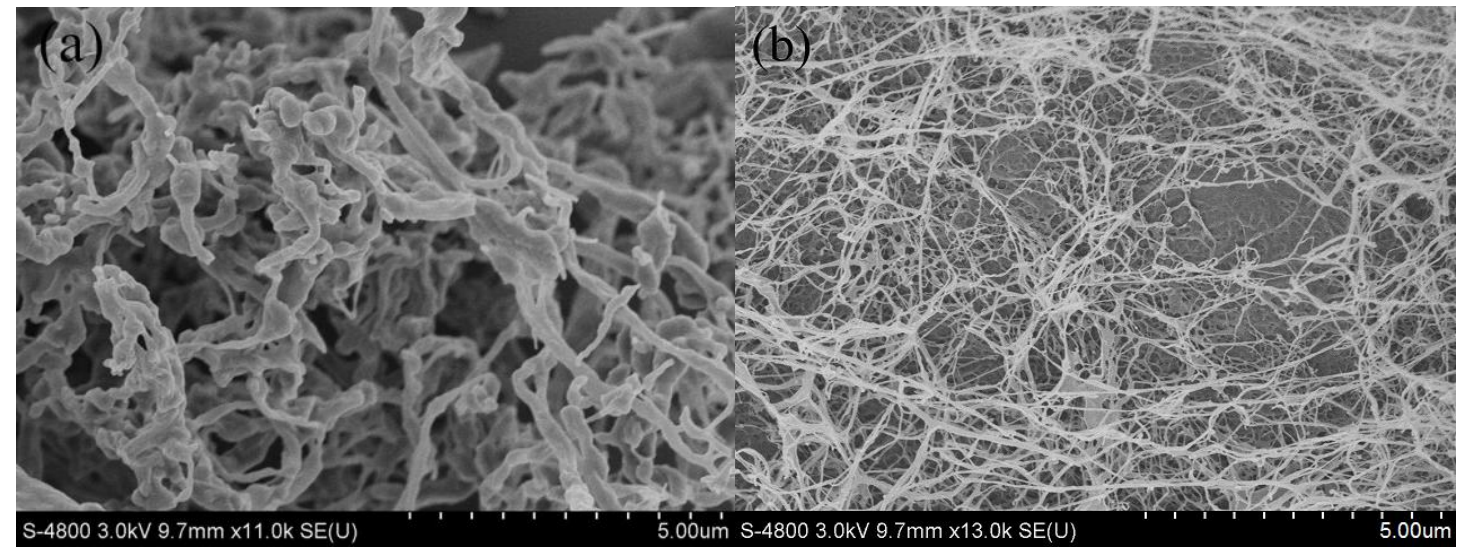

Fig. 5 SEM images of the xerogels of FA (a) and FAG (b).

TEM is another technique to characterize the morphology of gels. As shown in Fig. 6, FAG gel exhibits a nano-fibrous structure with a diameter of about $20 \mathrm{~nm}$, which is much thinner than FA gel $(20-60 \mathrm{~nm})$, and the network structure of FAG gel is obviously much denser than that of FA gel. The result is well in accordance with the SEM observation. 


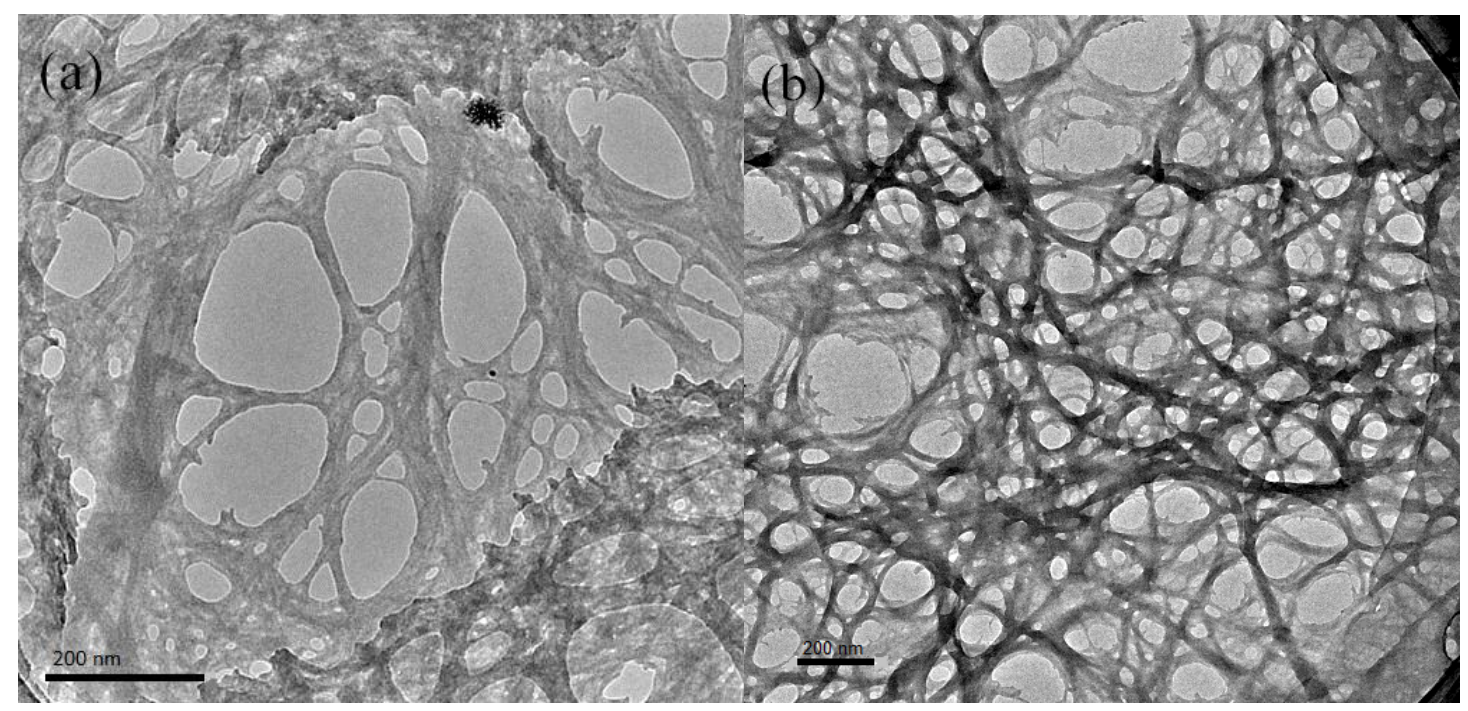

Fig. 6 TEM images of FA (a) and FAG (b) gels.

FA powder exhibits good crystallinity as shown in Fig. 7a. While, the crystalline ability has a considerable decrease after the formation of the gel (Fig. 7b, c) for the interactions between molecules destroy the symmetry and regularity of molecular chains. The diffraction peak intensity of FAG xerogel at about $2 \theta=20.5^{\circ}$ (Fig. $7 b, c$ ) is a little higher than that of FA xerogel, indicating a better ordered structure. FA is a low molecular weight gelator, so the fibers in the network structure of FA gel are stubby and inordered (Fig. 5a, 6a). While, from Fig. 5b, 6b, we could clearly see that the fibers become much thinner and denser with the addition of agar, and some long molecular chains play a supportive role in the structure. So it can be estimated that the agar molecules perhaps act as supports to induce FA molecules aggregate and interact with each other to form one-dimensional fibers, and then intertwine into network structures (Fig. 7d). ${ }^{34}$ Consequently, the structure of FAG hybrid gel own better regularity and crystallinity. 


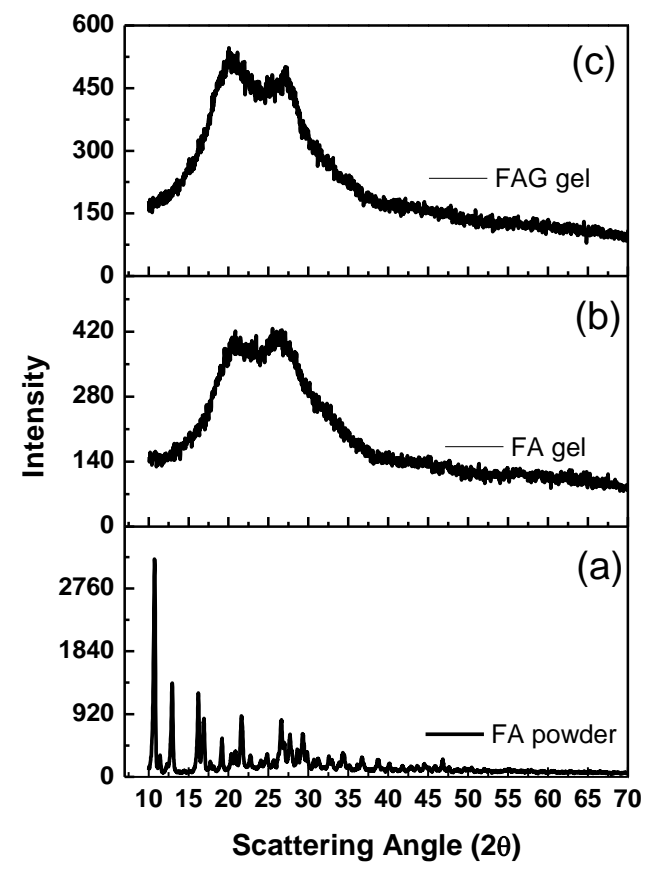

(d)

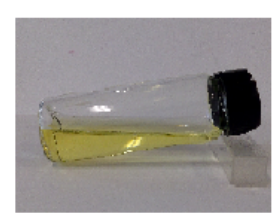

Mixed solution

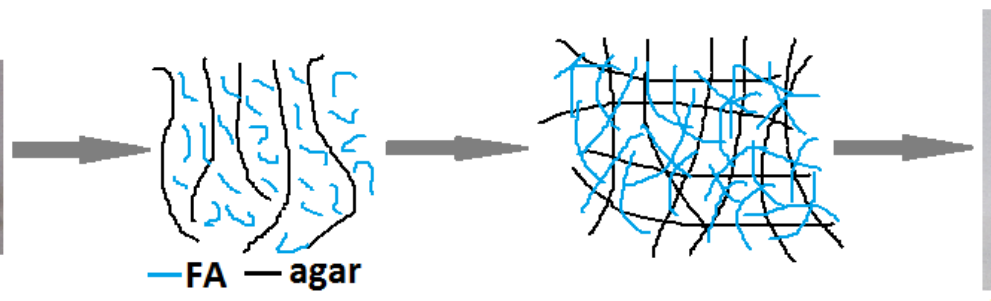

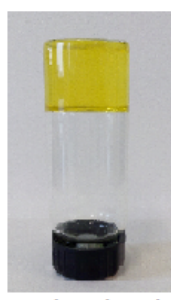

Hybrid gel

Fig. 7 XRD patterns of FA powder (a), FA xerogel (b) and FAG xerogel (c), and the schematic forming process of FAG hybrid gel (d).

Rheology is an obvious choice to investigate the deformation and flow of gel under the influence of an applied stress. For typical supramolecular gels, the storage modulus $\left(G^{\prime}\right)$, which reflects the resistance to deformation when a stress is applied, is always greater than loss modulus ( $\mathrm{G}^{\prime \prime}$, the contribution of viscous) over the entire range of frequencies in the linear viscoelastic region (LVR). ${ }^{35,36}$ The dynamic frequency sweep experiments of FA and FAG gels at $25{ }^{\circ} \mathrm{C}$ are shown in Fig. 8a. The $\mathrm{G}^{\prime}$ values are really higher than $\mathrm{G}^{\prime \prime}$, and both $\mathrm{G}^{\prime}$ and $\mathrm{G}^{\prime \prime}$ of the gels are invariant with frequency, indicating the formation of the gels. ${ }^{37,38}$ Gels prepared in the presence of agar show significantly larger G' and G" values than those without agar, which reveals 
the highly improvement of gel strength. The above XRD, FT-IR and UV-vis analyses have proved that agar involves in the self-assembly process of the gel and forms hydrogen bonds, $\pi-\pi$ interactions, etc. with FA molecules (Fig. 3, 4, 7). These interactions lead to changes of gel microstructure: thinner fibers, more branches and fiber-fiber entanglements and denser network structures (Fig. 5b and Fig. 6b). Consequently, the strength of FAG gel is enhanced. Furthermore, shear viscosity $v s$. shear rate in the LVR of the gels was also investigated( Fig. 8b). The viscosity of FAG hybrid gel is about 2 times higher than that of FA gel at the same shear rate. For both FA and FAG gels, the viscosities decreased as shear rate increased, which exhibits the non-Newtonian behavior following a shear-thinning property. At low shear rate, the destructed structures of the gels are capable of near-immediate recovery. While, with the increase of shear rate, the destruction rate of entangled structures will gradually exceed its recovery rate, when the shear rate reaches a certain value, the gel structures are almost completely destroyed and there is no time to form new entangled systems, so the viscosity reaches almost a constant minimum value. ${ }^{39}$ The shear-thinning property of FAG gels are essential for injectable materials. ${ }^{26}$
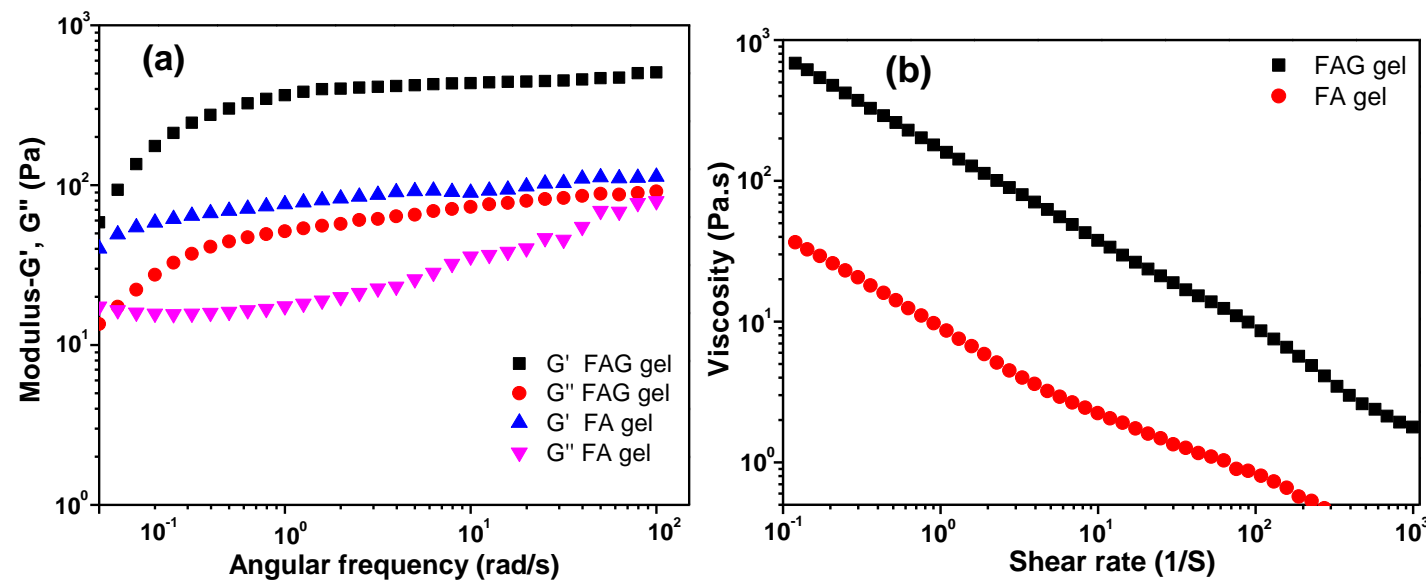

Fig. 8 Storage modulus $\left(\mathrm{G}^{\prime}\right)$ and loss modulus $\left(\mathrm{G}^{\prime \prime}\right)$ vs angular frequency of FA and 
FAG gels at $25{ }^{\circ} \mathrm{C}$ (a), shear viscosity vs shear rate of FA and FAG gels at $25{ }^{\circ} \mathrm{C}$ (b).

\section{Investigation of factors that affect FAG hybrid gels}

Owing to the weak and reversible driving forces of self-assembly, the supramolecular gel is facilely affected by many external factors, such as acid, base and salts, etc. Thus, the Tg and strength of FAG gel can be controlled by adjusting the conditions other than the agar. Here, we mainly investigated the impacts of $\mathrm{pH}$ and salt on the FAG gel.

We added different concentration of acid $(\mathrm{HCl})$ or base $(\mathrm{NaOH})$ solutions in the FA-agar mixture solutions to prepare FAG hybrid gels. As shown in Tab. 2, it could be clearly seen that the acid or base had exerted great impact on Tg. FA-agar mixture solution turned into gel at $\mathrm{pH}$ values between $2-10$ and it had a highest $\mathrm{Tg}, 37.5^{\circ} \mathrm{C}$ in neutral solution $(\mathrm{pH}=7)$. The pure FA solution could only turn into gels in the $\mathrm{pH}$ range of 4-8, and the $\mathrm{Tg}$ was all lower than that of FAG hybrid gels at the same $\mathrm{pH}$ value. At the same time, the impact of $\mathrm{pH}$ on gel strength was measured by putting a glass rod with a diameter of $0.442 \mathrm{~cm}$ on the surface of the gel, and applying pressure on the top of glass rod to measure the maximum pressure that the gel can endure. The result shown in Fig. S6 indicated that the gel strength of both FA and FAG gel was weakened with the addition of either acid or base. The transparence (see transmittance in Fig. 9) of FAG hybrid gels in acid condition is much lower than that in neutral or base state, the gel at $\mathrm{pH}=4$ is turbid, while it exhibits a transparent and weak state at $\mathrm{pH}=9$ (Fig. 9, inset). From Fig. S2a,b we could conclude that in acid condition, $-\mathrm{NH}_{2}$ and $-\mathrm{COOH}$ groups in FA become protonated, thus weakens the $\mathrm{H}$-bonding and $\pi-\pi$ 
interactions, and reduces the physical crosslink points in gel networks and then leads to the collapse of network structures,${ }^{40}$ so stubby and disorganized fibers are obtained ( Fig. S5a) and the gel becomes turbid. While, as a certain amount of base is added, the $-\mathrm{COOH}$ groups in FA and $-\mathrm{OH}$ groups in agar turn to $-\mathrm{COO}^{-}$and $-\mathrm{O}^{-} \mathrm{Na}^{+}$, so both FA and agar are more hydrophilic, and the number of fibers diminishes (Fig. S5b), causing more transparent gel with lower $\mathrm{Tg}$.

Tab. 2 Effect of $\mathrm{pH}$ values on the Tg of FA and FAG gels (Cagar is $0.13 \%$ (w/v))

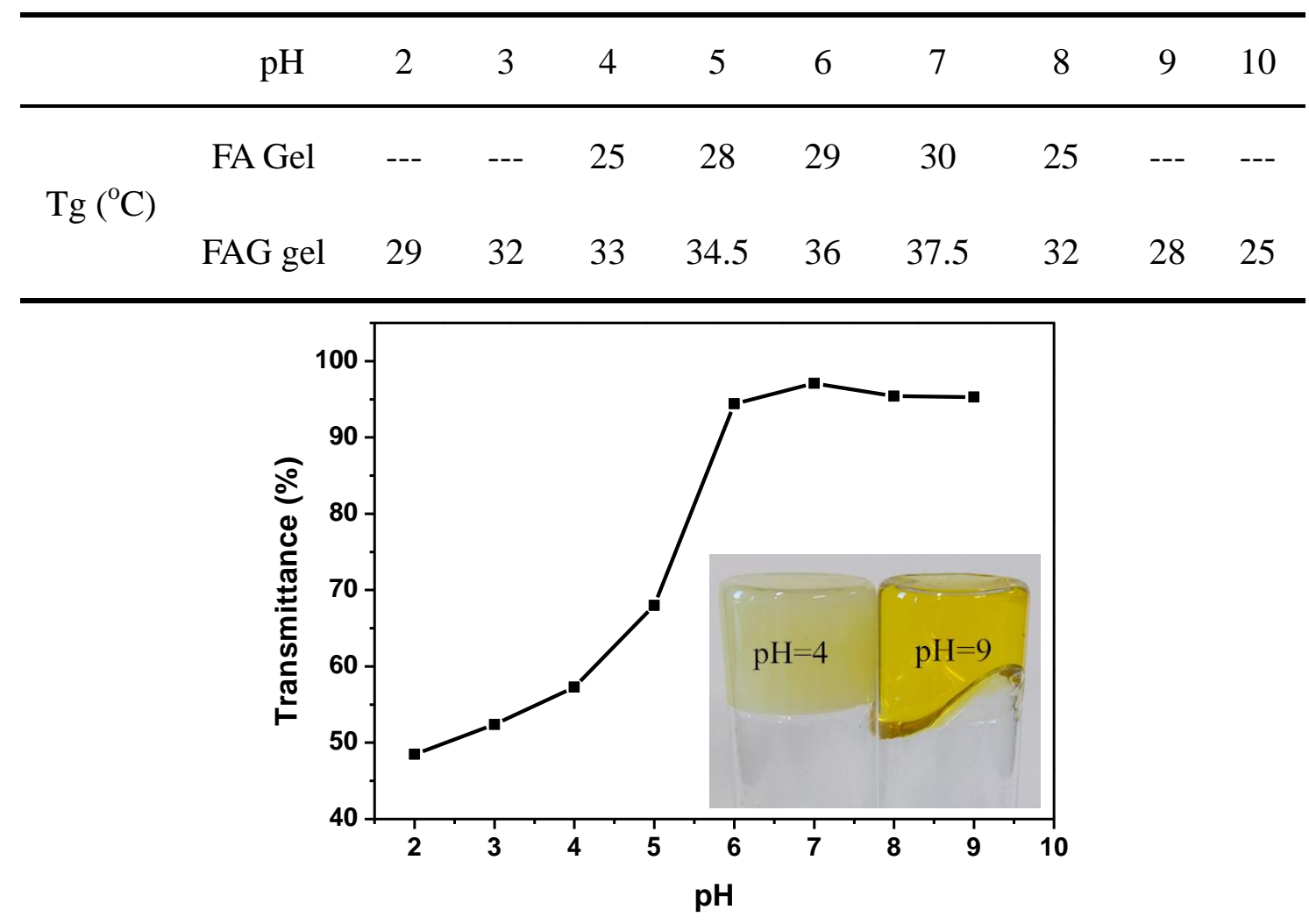

Fig. 9 Transmittance of FG hybrid gels at different $\mathrm{pH}$ values ( inset: FAG hybrid gel at $\mathrm{pH}=4$ and $\mathrm{pH}=9$ )

$\mathrm{NaCl}$ was chosen to investigate the effects of salt on the FAG gel and the results are shown in Fig. 10a. The Tg of FAG hybrid gels continuously increases with the increasing amount of $\mathrm{NaCl}$ and then decrease after the $\mathrm{NaCl}$ amount exceeds $40 \%$ (based on $0.2 \%$ (w/v) FA). The highest Tg of FAG hybrid gel reaches about $38.5^{\circ} \mathrm{C}$. 
So when the FAG gel is used as injectable material, the Tg could be adjusted by adding different amounts of $\mathrm{NaCl}$. Furthermore, the gel strength was measured with a glass rod as previously described. The maximum pressure that the FAG gel can be endure was shown in Fig. 10b, the FAG gel with $40 \% \mathrm{NaCl}$ can bear the maximum pressure, $8.286 \mathrm{kPa}$. The reason for the phenomenon is that $\mathrm{Na}^{+}$ions impact gel formation: within an appropriate proportion (0-40\%), molecular-ion interactions occur, and thus enhance the gel strength and Tg. While, when $\mathrm{NaCl}$ is above $40 \%$, extra $\mathrm{Na}^{+}$ ions produce charge shielding effects which obstruct the interactions of the positive and negative charges in gel systems, ${ }^{31}$ consequently, leading to the decrease of gel strength and Tg. Moreover, the gelation time (GT) could also be adjusted by changing the content of $\mathrm{NaCl}$, as exhibited in Tab. 3. The FAG gel including $40 \%$ of $\mathrm{NaCl}$ owns the shortest GT at $37{ }^{\circ} \mathrm{C}$, about $8.7 \mathrm{~min}$. Therefore, it is flexible to choose corresponding gel suitable for different applications in biomedical engineering when the FAG hybrid gels are applied as injectable gels.
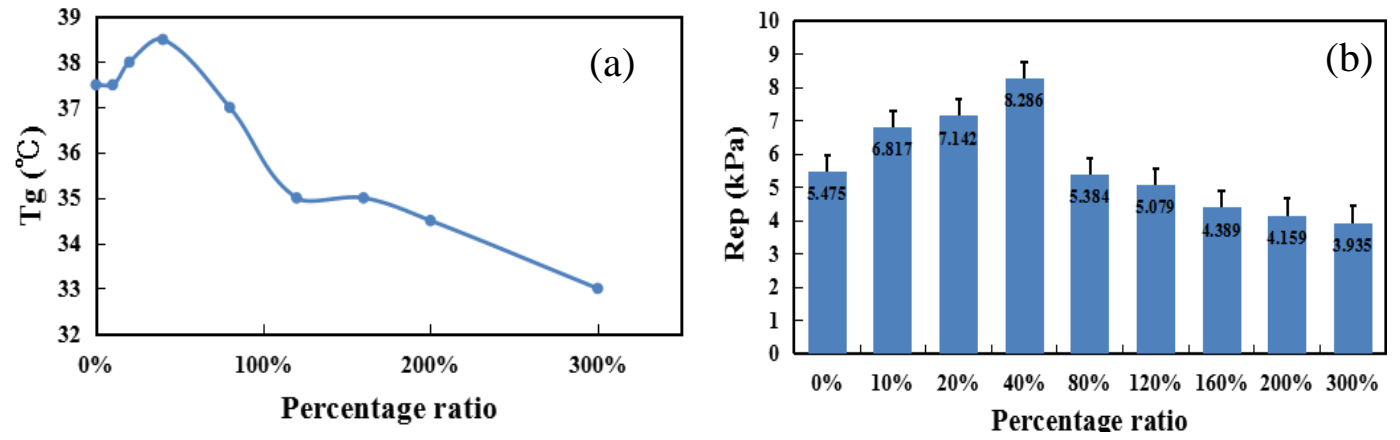

Fig. $10 \mathrm{Tg}$ (a) and resistance to external pressure (Rep) (b) of FAG gels containing different contents of $\mathrm{NaCl}$.

Tab. 3 GT of FAG hybrid gels with different percentage of $\mathrm{NaCl}$ at $37{ }^{\circ} \mathrm{C}$

$\begin{array}{lllll}\text { Percentage of } \mathrm{NaCl}(\%) & 0 & 10 & 20 & 40\end{array}$




$\begin{array}{lllll}\text { GT (min) } & 13.2 & 12.5 & 10.3 & 8.7\end{array}$

\section{Stability of FA and FAG gels}

The stability of the FAG gel is essential when it is considered for application in drug release. So we studied the stabilities of FA and FAG gels in different pH solutions. The tests were performed as follows: $10 \mathrm{~mL}$ of FA and FAG gels were placed in 20 $\mathrm{mL}$ of the solutions with the $\mathrm{pH}$ of $1,4,7$ and 10 , respectively. And then their remainder mass were measured at different times (each test was prepared in triplicate) $)^{41}$ for calculating the remaining mass fraction. The results (Fig. S7a-d) indicate that both FA and FAG gels suffer certain mass loss over the time. It is evidently that the gel entangled with agar shows much better stability than pure FA gel in each of $\mathrm{pH}$ solution and has lower mass loss. It is interesting to note the curves in Fig. 11 that the gels have the best stability under neutral condition and the worst stability in alkaline environment, which coincided well with the above results of $\mathrm{Tg}$.

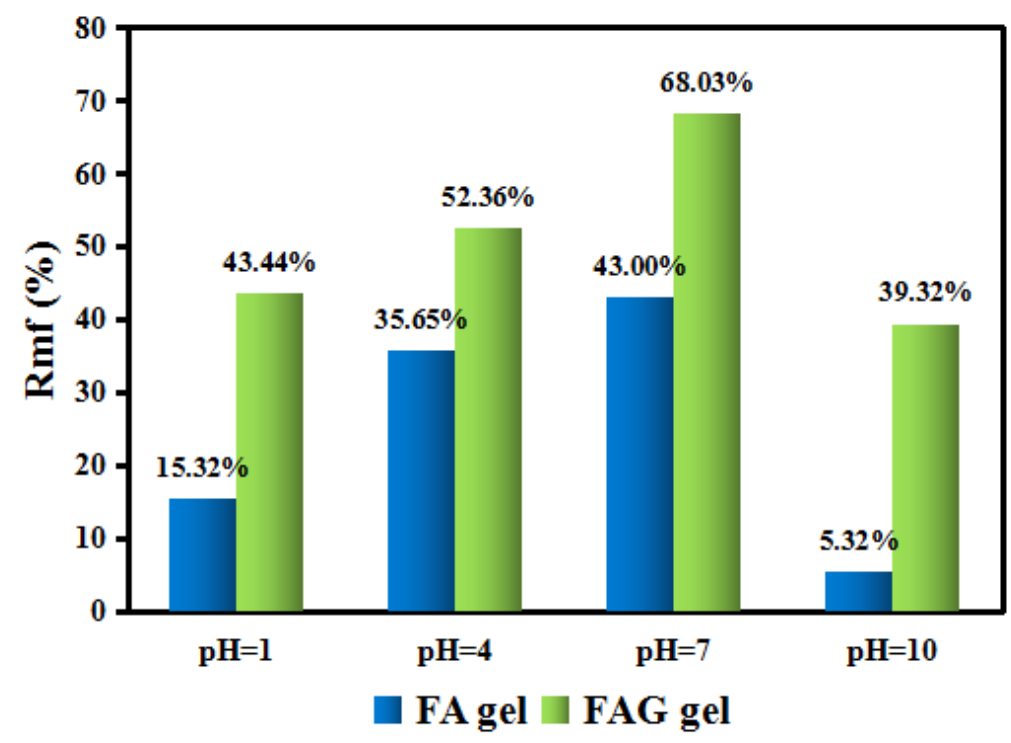

Fig. 11 The final remainder mass fraction (Rmf) of FA and FAG gels in different $\mathrm{pH}$ 
solutions after 6 hours.

\section{Release profile of RB contained FAG gels}

Folic acid, a kind of vitamin B, is indispensable for our body to produce new cells and repair DNA. Agar is an edible polysaccharide. DMSO is often employed as the vehicle control for both in vivo and in vitro as its wide solubilizing capability. ${ }^{42,43}$ Thus, as-prepared FAG hybrid gel is a type of non-toxic soft material. Furthermore, the self-healing property and shear-thinning behavior make such soft materials widely applicable in the fields of drug release. ${ }^{44,45}$ In this paper, Rhodamine B (RB), a kind of dye, was selected as a mimic model drug to investigate the drug release properties of the injectable FAG gel. The release profile of the RB loaded FAG gel is shown in Fig. 12. The release behavior could be divided into initial burst release, controlled release and equilibrium release periods. ${ }^{46,47}$ In the first two hours, both FA and FAG gels have undergone a burst release due to a great differential concentration between the medium and gels. More than $70 \%$ RB in FA gel diffuses into the medium in the first two hours, which suggests that the network structure of FA gel is loose. Compared with FA gel, the control release capability of FAG hybrid gel is much better, and the burst release amount within two hours is about $40 \%$ due to its dense network structure. The $\mathrm{RB}$ in FA gel reaches its release equilibrium after 10 hours, at which the accumulative release amount is about $87 \%$. While, FAG hybrid gel keeps a stable release in the period of 3-29 hours and reaches the release equilibrium after 30 hours. At that time, the accumulative release amount is about $86 \%$. Therefore, it is 
interesting to note that the release rate of $\mathrm{RB}$ exhibits a strong correlation to the structure and strength of the gels: RB molecules release faster from weaker gels than those from stronger gels. And thus, incorporation of agar makes FAG hybrid gel own better controlled release capacity and longer release time.

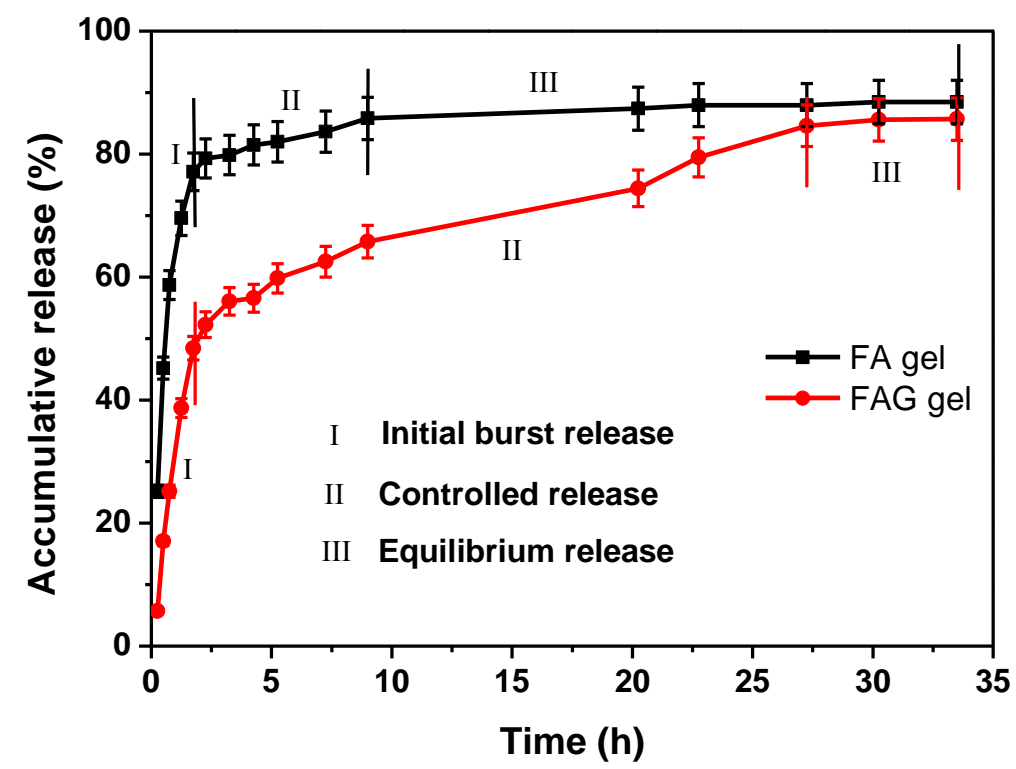

Fig. 12 Release profile of RB loaded FA gel and FAG gel in the release medium of normal saline $(0.9 \% \mathrm{NaCl})$.

\section{Conclusions}

In conclusion, we have developed new injectable hybrid gels in this paper by successfully incorporating a kind of biological macromolecule, agar, into FA gels. The FT-IR spectra of the FAG hybrid gels indicate that agar molecules involve in the process of self-assembly via forming hydrogen bonds with FA and create a denser network structure with thinner fibers. In consequence, the strength of FAG hybrid gel is greatly improved. For FAG hybrid gel, both of the storage modulus ( $\left.\mathrm{G}^{\prime}\right)$ and loss modulus $\left(G^{\prime \prime}\right)$ exhibit sharp increases. The shear viscosity is about 2 times higher than 
that of FA gel and shows a shear-thinning behavior. Furthermore, the gelation temperature of FAG hybrid gels could be adjusted to about body temperature $\left(37^{\circ} \mathrm{C}\right)$, and the gelation time is adjustable by adding different amounts of $\mathrm{NaCl}$. These properties make FAG gel ideal injectable materials for applications in drug release. Compared with FA gel, the RB loaded injectable FAG hybrid gel shows better sustained release properties, such as much lower initial burst and longer release time. Therefore, injectable FAG hybrid gel is a potential injectable material for applications in drug release or tissue engineering as scaffolds.

\section{Acknowledgement}

This work was supported by the National Science Foundation of China (51573126).

\section{References}

1. W. Edwards and D. K. Smith, Dynamic Evolving Two-Component Supramolecular Gels-Hierarchical Control over Component Selection in Complex Mixtures, J. Am. Chem. Soc., 2013, 135, 5911-5920.

2. X. Z. Yan, D. H. Xu, X. D. Chi, J. Z. Chen, S. Y. Dong, X. Ding, Y. H. Yu and F. H. Huang, A Multiresponsive, Shape-Persistent, and Elastic Supramolecular Polymer Network Gel Constructed by Orthogonal Self-Assembly, Adv. Mater, 2012, 24, 362-369.

3. Z. H. Qi and C. A. Schalley, Exploring Macrocycles in Functional Supramolecular 
Gels: From Stimuli Responsiveness to Systems Chemistry, Acc. Chem. Res., 2014, 47, 2222-2233.

4. H. Maeda, Anion-Responsive Supramolecular Gels, Chem. Eur. J, 2008, 14, $11274-11282$.

5. N. M. Sangeetha and U. Maitra, Supramolecular gels: Functions and uses, Chem.Soc. Rev., 2005, 34, 821-836.

6. S. Seiffert and J. Sprakel, Physical chemistry of supramolecular polymer networks, Chem. Soc. Rev., 2012, 41, 909-930.

7. J. W. Steed, Supramolecular gel chemistry: developments over the last decade, Chem. Commun., 2011, 47, 1379-1383.

8. A. Noro, M. Hayashi and Y. S. Matsushita, Design and properties of supramolecular polymer gels, Soft Matter, 2012, 8, 6416-6429.

9. L. A. Estroff, L. Addadi, S. Weiner and A. D. Hamilton, An organic hydrogel as a matrix for the growth of calcite crystals $\dagger$, Org. Biomol. Chem., 2004, 2, 137-141.

10. L. E. Buerklea and S. J. Rowan, Supramolecular gels formed from multi-component low molecular weight species, Chem. Soc. Rev., 2012, 41, 6089-6102.

11. J. Y. Zhan, M. M. Zhang, M. Zhou, B. Liu, D. Chen, Y. Y. Liu, Q. Q. Chen, H. Y. Qiu and S. C. Yin, A Multiple-Responsive Self-Healing Supramolecular Polymer Gel Network Based on Multiple Orthogonal Interactions, Macromol. Rapid Commun., 2014, 35, 1424-1429.

12. G. H. Deng, C. M. Tang, F. Y. Li, H. F. Jiang and Y. M. Chen, Covalent 
Cross-Linked Polymer Gels with Reversible Sol-Gel Transition and Self-Healing Properties, Macromolecules, 2010, 43, 1191-1194.

13. S. Matsumoto, S. Yamaguchi, S. Ueno, H. Komatsu, M. Ikeda, K. Ishizuka, Y. Iko, K. V. Tabata, H. Aoki, S. Ito, H. Noji and I. Hamachi, Photo Gel-Sol/Sol-Gel Transition and Its Patterning of a SupramolecularHydrogel as Stimuli-Responsive Biomaterials, Chem. Eur. J., 2008, 14, 3977 - 3986.

14. G. A. Silva, C. Czeisler, K. L. Niece, E. Beniash, D. A. Harrington, J. A. Kessler and S. I. Stupp, Selective Differentiation of Neural Progenitor Cells by High-Epitope Density Nanofibers, Science, 2004, 303, 1352-1355.

15. A. Klaewklod, V. Tantishaiyakul, N. Hirun, T. Sangfai and L. Li, Characterization of supramolecular gels based on $\beta$-cyclodextrin and polyethyleneglycol and their potential use for topical drug delivery, Materials Science and Engineering C, 2015, $50,242-250$.

16. T. Tu, W. W. Fang and Z. M. Sun, Visual-Size Molecular Recognition Based on Gels, Adv. Mater., 2013, 25, 5304-5313.

17. Y. J. Adhia, T. H. Schloemer, M. T. Perez and A. J. McNeil, Using polymeric additives to enhance molecular gelation: impact of poly(acrylic acid) on pyridine-based gelators $\dagger$, Soft Matter, 2012, 8, 430-434.

18. P. Chakraborty, B. Roy, P. Bairi and A. K. Nandi, Improved mechanical and photophysical properties of chitosan incorporated folic acid gel possessing the characteristics of dye and metal ion absorption†, J. Mater. Chem., 2012, 22, 20291-20298. 
19. "Dietary supplement fact sheet: Folate". Health Information. Office of Dietary Supplements, US National Institutes of Health.

20. Pommerville, Jeffrey C, Alcamo's Fundamentals of Microbiology: Body Systems. Jones \&Bartlett Publishers. P. 511. ISBN 9780763787127.

21. P. Y. Xing, X. X. Chu, M. F. Ma, S. Y. Li and A. Y. Hao, Supramolecular gel from folic acid with multiple responsiveness, rapid self-recovery and orthogonal self-assemblies $\dagger$, Phys. Chem. Chem. Phys., 2014, 16, 8346-8359.

22. P. Chakraborty, P. Bairi, B. Roy and A. K. Nandi, Improved Mechanical and Electronic Properties of Co-assembled Folic Acid Gel with Aniline and Polyaniline, ACS Appl. Mater. Interfaces, 2014, 6, 3615-3622.

23. A. Chenite, C. Chaput, D. Wang, C. Combes, M.D. Buschmann, C.D. Hoemann, J.C Leroux, B.L. Atkinson, F. Binette and A. Selmani, Novel injectable neutral solutions of chitosan form biodegradable gels in situ, Biomaterials, 2000, 21, $2155-2161$

24. S. Yasmeen, M. K. Lo, S. Bajracharya and M. Roldo, Injectable Scaffolds for Bone Regeneration, Langmuir, 2014, 30, 12977-12985.

25. N. Q. Tran, Y. K. Joung, E. Lih, K. M. Park and K. D. Park, Supramolecular Hydrogels Exhibiting Fast In Situ Gel Forming and Adjustable Degradation Properties, Biomacromolecules, 2010,11, 617-625.

26. J. Yu, W. Ha, J.-N. Sun and Y.-P. Shi, Supramolecular Hybrid Hydrogel Based on Host-Guest Interaction and Its Application in Drug Delivery, ACS Appl. Mater. Interfaces, 2014, 6, 19544-19551. 
27. S. J. Weinstein, T. J. Hartman, R. Stolzenberg-Solomon, P. Pietinen, M. J. Barrett, P. R. Taylor, J. Virtamo and D. Albanes, Null Association between Prostate Cancer and Serum Folate, Vitamin B6, Vitamin B12, and Homocysteine, AACR, 2003, 12, $1271-1272$.

28. J. Zhang, S. Rana, R.S. Srivastava and R.D.K. Misra, On the chemical synthesis and drug delivery response of folate receptor-activated, polyethylene glycol-functionalized magnetite nanoparticles, Acta Biomaterialia, 2008, 4, 40-48.

29. X. Y. Yang, G. X. Zhang and D. Q. Zhang, Stimuli responsive gels based on low molecular weight gelators, J. Mater. Chem., 2012, 22, 38-50.

30. F. Aparicio, E. Matesanz and L. Sánchez, Cooperative self-assembly of linear organogelators. Amplification of chirality and crystal growth of pharmaceutical ingredientsw, Chem. Commun., 2012, 48, 5757-5759.

31. Y. Y. Li, J. Liu, G. Y. Du, H. Yan, H. Y. Wang, H. C. Zhang, W. An, W. J. Zhao, T. Sun, F. Xin, L. Kong, Y. M. Li, A. Y. Hao and J. C. Hao, Reversible Heat-Set Organogel Based on Supramolecular Interactions of -Cyclodextrin in N,N-Dimethylformamide, J. Phys. Chem. B, 2010, 114, 10321-10326.

32. X. Y. Liu and P. D. Sawant, Micro/Nanoengineering of the Self-Organized Three-Dimensional Fibrous Structure of Functional Materials, Angew. Chem. Int. Ed., 2002, 41, 3641-3645.

33. J. L. Li and X. Y. Liu, Architecture and engineering of a supramolecular functional material by manipulating the nanostructure of fiber network, Appl. Phys. Lett., $2005,87,113103$. 
34. M. Numata, K. Sugiyasu, T. Kishida, S. Haraguchi, N. Fujita, S. M. Park, Y. J. Yun, B. H. Kim and S. Shinkai, Creation of polynucleotide-assisted molecular assemblies in organic solvents: general strategy toward the creation of artificial DNA-like nanoarchitectures, Org. Biomol. Chem., 2008, 6, 712-718.

35. M. O. M. Piepenbrock, G. O. Lloyd, N. Clarke and J. W. Steed, Metal- and Anion-Binding Supramolecular Gels, Chem. Rev., 2010, 110, 1960-2004.

36. M. M. Zhang, D. H. Xu, X. Z. Yan, J. Z. Chen, S. Y. Dong, B. Zheng and F. H. Huang, Self-Healing Supramolecular Gels Formed by Crown Ether Based Host-Guest Interactions**, Angew. Chem. Int. Ed., 2012, 51, 7011-7015.

37. G. C. Yu, X. Z. Yan, C. Y. Han and F. H. Huang, Characterization of supramolecular gels, Chem. Soc. Rev., 2013, 42, 6697-6722.

38. X. Q. Cai, K. Q. Liu, J. L. Yan, H. L. Zhang, X. Y. Hou, Z. Liu and Y. Fang, Calix[4]arene-based supramolecular gels with unprecedented rheological properties $\dagger$, Soft Matter, 2012, 8, 3756-3761.

39. C. B. Rodell, A. L. Kaminski and J. A. Burdick, Design of Network Properties in Guest-Host Assembled and Shear-Thinning Hyaluronic Acid Hydrogels, Biomacromolecules, 2013, 14, 4125-4134.

40. S. L. Zhou, S. Matsumoto, H.-D. Tian, H. Yamane, A. Ojida, S. Kiyonaka and I. Hamachi, pH-Responsive Shrinkage/Swelling of a Supramolecular Hydrogel Composed of Two Small Amphiphilic Molecules, Chem. Eur. J., 2005, 11, 1130-1136.

41. B. Buchs, W. Fieber, F. Vigouroux-Elie, N. Sreenivasachary, J.-M. Lehn and 
A.Herrmann, Release of bioactive volatiles from supramolecular hydrogels: influence of reversible acylhydrazone formation on gel stability and volatile compound evaporation†, Org. Biomol. Chem., 2011, 9, 2906-2919.

42. S. C.Modesitt and S. J. Parsons, In vitro and in vivo histone deacetylase inhibitor therapy with vorinostat and paclitaxel in ovarian cancer models: Does timing

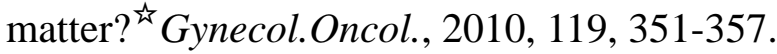

43. V. K. Gupta, Y. You, J. C. Li, A. Klistorner and S. L. Graham, Protective Effects of 7,8-Dihydroxyflavone on Retinal Ganglion and RGC-5 Cells Against Excitotoxic and Oxidative Stress, J Mol Neurosci, 2013, 49, 96-104.

44. H. D. Lu, M. B. Charati, I. L. Kim and J. A. Burdick, Injectable shear-thinning hydrogels engineered with a self-assembling Dock-and-Lock mechanism, Biomaterials, 2012, 33, 2145-2153.

45. C. T. S. Wong Po Foo, J. S. Lee, W. Mulyasasmita, A. Parisi-Amon and S. C. Heilshorn, Two-component protein-engineered physical hydrogels for cell encapsulation, PNAS, 2009, 106, 22067-22072.

46. M. Rodrigues, A. C. Calpena, D. B. Amabilino, M. L. Garduño- Ramírez and L. Pérez-García, Supramolecular gels based on a gemini imidazolium amphiphile as molecular material for drug delivery†, J. Mater. Chem. B, 2014, 2, 5419-5429.

47. S. Sutton, N. L. Campbell, A. I. Cooper, M. Kirkland, W. J. Frith and D. J. Adams, Controlled Release from Modified Amino Acid Hydrogels Governed by Molecular Size or Network Dynamics, Langmuir, 2009, 25, 10285-10291. 